Proyecciones

Vol. 24, $\mathrm{N}^{o}$ 3, pp. 277-286, December 2005.

Universidad Católica del Norte

Antofagasta - Chile DOI: 10.4067/S0716-09172005000300006

\title{
AN IMPROVEMENT OF J. RIVERA-LETELIER RESULT ON WEAK HYPERBOLICITY ON PERIODIC ORBITS FOR POLYNOMIALS
}

\author{
FELIKS PRZYTYCKI * \\ Polish Academy of Sciences, Polonia \\ Received : January 2005. Accepted : November 2005
}

\begin{abstract}
We prove that for $f: \overline{\mathscr{C}} \rightarrow \overline{\mathbb{C}}$ a rational mapping of the Riemann sphere of degree at least 2 and $\Omega$ a simply connected immediate basin of attraction to an attracting fixed point, if $\left|\left(f^{n}\right)^{\prime}(p)\right| \geq C n^{3+\xi}$ for constants $\xi>0, C>0$ all positive integers $n$ and all repelling periodic points $p$ of period $n$ in Julia set for $f$, then a Riemann mapping $R: \mathbb{D} \rightarrow \Omega$ extends continuously to $\bar{D}$ and $\operatorname{Fr} \Omega$ is locally connected. This improves a result proved by J. Rivera-Letelier for $\Omega$ the basin of infinity for polynomials, and $5+\xi$ rather than $3+\xi$.
\end{abstract}

* Supported by Polish KBN grant 2P03A 03425 
We prove the following

Theorem 1. Let $f$ be a polynomial of 1 complex variable of degree at least 2, with connected Julia set. Suppose there are constants $C>0$ and $\xi>0$ such that for every repelling periodic point $p$ in the complex plane $\mathbb{C}$ of period $n$,

$$
\left|\left(f^{n}\right)^{\prime}(p)\right| \geq C n^{3+\xi}
$$

Then a Riemann map $R: \overline{\mathbb{C}} \backslash \overline{\mathbb{D}} \rightarrow \overline{\mathbb{C}} \backslash K(f)$ from the complement of the closure of the unit disc $\mathbb{D}$ to the complement of the filled-in Julia set in the Riemann sphere, extends continuously to $\overline{\mathscr{C}} \backslash \mathbb{D}$. In particular Julia set is locally connected and there are no Cremer periodic orbits. $\mathrm{Cn} n^{5+\xi}$

In $[\mathrm{R}]$ Juan Rivera-Letelier proved this under the assumption $\left|\left(f^{n}\right)^{\prime}(p)\right| \geq$

The same strategy proves in fact a stronger theorem below, in the setting of [P2], including the case of an arbitrary simply connected immediate basin of attraction to a periodic sink for a rational map of $\overline{\mathscr{C}}$.

Theorem 2. Let $f$ be a rational mapping on the Riemann sphere $\overline{\mathbb{C}}$ of degree at least 2 and let $\Omega$ be a simply connected immediate basin of attraction to an attracting fixed point. Suppose that $\left(^{*}\right)$ holds for all repelling periodic points $p$ in Julia set for $f$. Then any Riemann map $R: \mathbb{D} \rightarrow \Omega$ extends continuously to $\bar{D}$ and $\operatorname{Fr} \Omega$ is locally connected.

Most part of our proof of Theorems 1 and 2 follows $[R]$. The proof of Theorem 1 uses an invariant measure of maximal entropy. However the right measure to use in more general situations, like in Theorem 2 , is an $f$-invariant measure $\omega$ equivalent to a harmonic measure on $\operatorname{Fr} \Omega$ viewed from $\Omega$; it coincides with the measure of maximal entropy in the case of the basin of $\infty$ for polynomials.

In the situation of Theorem 2 there is however a technical difficulty, namely proving the existence of an expanding repeller $X$ in $\operatorname{Fr} \Omega$, such that in particular the topological entropy of $\left.f\right|_{X}$ is arbitrarily close to the measure theoretical entropy $h_{\omega}(f)$, in consequence such that Hausdorff dimension $\operatorname{HD}(X)$ is arbitrarily close to $\operatorname{HD}(\omega)=1$, see Lemma 3. This fact is a strengthening of the theorem on the density of periodic points in $\operatorname{Fr} \Omega$, see [PZ]. The proof can be obtained as in [PZ] with the use of Pesin-Katok theory and is omitted here. We devote a separate short paper [P4] to it. In 
the situation of Theorem 1 the existence of $X$ is also needed in the proof, but this case is easier (see the references in $[R]$ ).

Proof of Theorem 1 (and analogously Theorem 2) reduces to checking the summability assumption in the following standard

Lemma 1 , see $[\mathrm{R}]$. Let $w_{0} \in \mathbb{C} \backslash K(f)$ and $\omega_{n}, n=1,2, \ldots$ be an increasing sequence of positive real numbers such that $\sum_{n=1}^{\infty} \omega_{n}^{-1}<\infty$. If for every $w \in f^{-n}\left(w_{0}\right)$ we have $\left|\left(f^{n}\right)^{\prime}(w)\right| \geq \omega_{n}$, then the Riemann map $R$ extends continuously to $\overline{\mathbb{C}} \backslash \mathbb{D}$.

Definitions. We call a closed set $X \subset J(f)$ an expanding repeller if $f(X) \subset X$ the map $f$ restricted to $X$ is open, topologically mixing and expanding.

Here expanding means that there exist $C>0$ and $\lambda>1$, called an expanding constant, such that for every $x \in X$ we have $\left|\left(f^{n}\right)^{\prime}(x)\right| \geq C \lambda^{n}$. The property that $\left.f\right|_{X}$ is open is equivalent to the existence of a neighbourhood $U$ of $X$ in $\mathscr{C}$, called a repelling neighbourhood, such that every forward $f$-trajectory $x, f(x), \ldots f^{n}(x), \ldots$ staying in $U$ must be contained in $X$, see for example [PU1, Ch.5]. This easily implies that if $\left\{x, f(x), \ldots f^{n}(x)\right\} \subset U$ then $\left|\left(f^{n}\right)^{\prime}(x)\right| \geq C \lambda^{n}$, maybe for a constant $C$ bigger than before and $U$ a smaller neighbourhood of $X$.

Let $\lambda_{n}, n=1,2, \ldots$ be an increasing sequence of positive real numbers such that for every $n$, every repelling periodic point $p$ of period $n$ has the multiplier $\left(f^{n}\right)^{\prime}(p)$ of absolute value at least $\lambda_{n}$.

In the sequel $C$ will denote various positive constants which can change even in one consideration.

Lemma 2, see $[\mathrm{R}]$. Let $f$ be a polynomial of 1 complex variable of degree at least 2 . Let $X \subset J(f)$ be an expanding repeller of positive Hausdorff dimension, $\operatorname{HD}(X)>0$, and $\lambda$ be its expanding constant. Then there is $U$, a repelling neighbourhood of $X$, a "base point" $w_{0} \in \mathbb{C} \backslash K(f)$ and a constant $C>0$ such that the following holds.

For every $\varepsilon>0$ for every $n$ large enough there exists an integer $\ell=\ell(n)$ satisfying $0 \leq \ell \leq(1 /(\mathrm{HD}(X) \ln \lambda)+\varepsilon) \ln n$, and there exists $x=x(n) \in$ $f^{-\ell}\left(w_{0}\right)$ satisfying $x, \ldots, f^{\ell}(x) \in U$, such that for every $z \in f^{-n}(x)$

$$
\left|\left(f^{n+\ell}\right)^{\prime}(z)\right| \geq C \lambda_{n+\ell} .
$$

Sketch of Proof. This Lemma in a slightly different formulation was proved in $[R]$ and in a more rough version in [PRS1]. See also [PRS2, §2] 
and [P-Kyoto]. The idea is first to find $\hat{x} \in X$, a safe point, that is

$$
\hat{x} \notin\left(\bigcap_{k=1}^{\infty} \bigcup_{n \geq k} B\left(f^{2 n}(\operatorname{Crit}(f)) \cup f^{2 n+1}(\operatorname{Crit}(f)), n^{-a}\right)\right) \cup \bigcup_{n \geq 1} f^{n}(\operatorname{Crit}(f))
$$

for an arbitrarily fixed $a>1 / \operatorname{HD}(X)$. The latter inequality assures the existence of $\hat{x}$. Here $\operatorname{Crit}(f)$ denotes the set of all $f$-critical points in $\mathbb{C}$.

Fix an arbitrary point $\hat{w} \in X$ and $r_{0}>0$ such that $B^{\prime}:=B\left(\hat{w}, r_{0}\right)$ is well inside $U$ and choose an arbitrary $w_{0} \in B^{\prime} \backslash K$ as a base point.

Let $\ell$ be a minimal time such that a component $V$ of $f^{-\ell}\left(B^{\prime}\right)$ intersecting $X$ is in $B^{\prime \prime}:=B\left(\hat{x}, \delta n^{-a}\right)$ ), where $0<\delta<<1$ is a constant. By construction $f^{\ell}$ is univalent on $V$ and has bounded distortion. Denote the branch of $f^{-\ell}$ leading $B^{\prime}$ to $V$ by $F_{1}$.

(More precisely, $F_{1}$ can be constructed in two steps. First, let $k$ be the smallest integer such that $f^{k}$ maps $B^{\prime \prime}$ to a boundedly distorted large disc $B^{\prime \prime \prime}$. Denote the branch of $f^{-k}$ leading $B^{\prime \prime \prime}$ to $B^{\prime \prime}$ by $F_{1}^{\prime}$. Next using the topological transitivity of $f$ on $X$ we find a branch $F_{1}^{\prime \prime}$ of $f^{-M}$ on $B^{\prime}$ mapping it in $B^{\prime \prime \prime}$, where $M$ is bounded independently of $n$. We define $F_{1}:=F_{1}^{\prime} \circ F_{1}^{\prime \prime}$ and $\ell=k+M$.)

Each branch $F_{2}$ of $f^{-n}$ on $B\left(\hat{x}, n^{-a}\right)$, can be composed with $F_{3}$ being the composition of at most $N$ branches of $f^{-1}$ for $N$ bounded independently of $n$, so that $F_{3} \circ F_{2}$ maps $B\left(\hat{x}, \delta n^{-a}\right)$ deep in $B^{\prime}$. Then $F=F_{3} \circ F_{2} \circ F_{1}$, a branch of $f^{-(n+\ell+N)}$, maps $B^{\prime}$ deep in itself, so $F\left(B^{\prime}\right)$ contains a periodic point $p$ of period $n+\ell+N$.

Finally replace $\hat{x} \in J(f)$ by $x \in V \backslash K(f)$ such that $f^{\ell}(x)=w_{0}$. For $z=F_{2}(x)$, since $\left|\left(f^{n+\ell+N}\right)^{\prime}\left(F_{3}(z)\right)\right| /\left|\left(f^{n+\ell+N}\right)^{\prime}(p)\right|$ is bounded by a distortion constant, we get

$$
\left|\left(f^{n+\ell}\right)^{\prime}(z)\right|=\left|\left(f^{n+\ell+N}\right)^{\prime}\left(F_{3}(z)\right)\right| \cdot\left|F_{3}^{\prime}(z)\right| \geq C \lambda_{n+\ell+N} \geq C \lambda_{n+\ell} .
$$

QED

Proof of Theorem 1. Let $X$ and other constants be as in Lemma 2 . Let $\beta_{2} \geq \beta_{1}>1$ be constants such that for all $k$ large enough and all $y$ such that $y, \ldots, f^{k}(y) \in U$ we have $\beta_{1}^{k} \leq\left|\left(f^{k}\right)^{\prime}(y)\right| \leq \beta_{2}^{k}$.

Consider an arbitrary $w_{n} \in f^{-n}\left(w_{0}\right)$. Join $x=x(n)$ to $w_{0}$ by a hyperbolic geodesic $\gamma=\gamma_{n}$ in $\mathbb{C} \backslash K(f)$. Let $x_{n}$ be the end of the component of $f^{-n}\left(\gamma_{n}\right)$ having one end at $w_{n}$, different from $w_{n}$. Then we write

$$
\left|\left(f^{n}\right)^{\prime}\left(w_{n}\right)\right|=\left|\left(f^{n}\right)^{\prime}\left(x_{n}\right)\right| \frac{\left|\left(f^{n}\right)^{\prime}\left(w_{n}\right)\right|}{\left|\left(f^{n}\right)^{\prime}\left(x_{n}\right)\right|} .
$$


By Lemma 2 we have

$$
\left|\left(f^{n}\right)^{\prime}\left(x_{n}\right)\right|=\left|\left(f^{n+\ell}\right)^{\prime}\left(x_{n}\right)\right| \cdot\left|\left(f^{\ell}\right)^{\prime}(x)\right|^{-1} \geq C \lambda_{n+\ell} \beta_{2}^{-\ell} .
$$

Denote $\tilde{w}_{0}=R^{-1}\left(w_{0}\right), \tilde{w}_{n}=R^{-1}\left(w_{n}\right), \tilde{x}=R^{-1}(x)$ and $\tilde{x}_{n}=R^{-1}\left(x_{n}\right)$.

We have

$$
\frac{\left|\left(f^{n}\right)^{\prime}\left(w_{n}\right)\right|}{\left|\left(f^{n}\right)^{\prime}\left(x_{n}\right)\right|}=\frac{\left|\left(R^{-1} \circ f^{n}\right)^{\prime}\left(w_{n}\right)\right|}{\left|\left(R^{-1} \circ f^{n}\right)^{\prime}\left(x_{n}\right)\right|} \frac{\left|R^{\prime}\left(\tilde{w}_{0}\right)\right|}{\left|R^{\prime}(\tilde{x})\right|}=\frac{\left|\left(f^{-n} \circ R\right)^{\prime}(\tilde{x})\right|}{\left|\left(f^{-n} \circ R\right)^{\prime}\left(\tilde{w}_{0}\right)\right|} \cdot \frac{\left|R^{\prime}\left(\tilde{w}_{0}\right)\right|}{\left|R^{\prime}(\tilde{x})\right|}=I \cdot I I,
$$

where $f^{-n}$ is the branch leading $x_{0}$ to $x_{n}$ and $w_{0}$ to $w_{n}$.

Note that $|\tilde{x}|-1 \geq C d^{-\ell}$, where $C$ depends only on $\left|\tilde{w}_{0}\right|$. We estimate the fraction I by Koebe Distortion Lemma. Namely there is a constant $C_{\mathrm{K}}$ depending only on $\tilde{w}_{0}$ such that

$$
I \geq C_{\mathrm{K}}(|\tilde{x}|-1) \geq C C_{\mathrm{K}} d^{-\ell} .
$$

We have also, denoting $g(z)=z^{d}$, using $R g^{\ell}=f^{\ell} R$,

$$
I I \geq\left|\left(f^{\ell}\right)^{\prime}(x)\right| \cdot\left|\left(g^{\ell}\right)^{\prime}(\tilde{x})\right|^{-1} \geq C d^{-\ell} \beta_{1}^{\ell} .
$$

In conclusion

$$
\left|\left(f^{n}\right)^{\prime}\left(w_{n}\right)\right| \geq C \lambda_{n} \beta_{2}^{-\ell} d^{-2 \ell} \beta_{1}^{\ell} .
$$

Invoking the estimate of $\ell$ we get

$$
\begin{gathered}
\left|\left(f^{n}\right)^{\prime}\left(w_{n}\right)\right| \geq C \lambda_{n} \beta_{2}^{-\ell} \beta_{1}^{\ell} d^{-2(1 /(\ln \lambda) \operatorname{HD}(X)+\varepsilon) \ln n} \\
\geq C \lambda_{n}\left(\beta_{1} / \beta_{2}\right)^{\ell} n^{-2(1 /(\ln \lambda) \operatorname{HD}(X)+\varepsilon) \ln d}
\end{gathered}
$$

By Pesin-Katok theory, applied to the measure of maximal entropy equal to $\ln d$, there exists $X$ and its repelling neighbourhood $U$, such that $\beta_{1} \geq d-\varepsilon$ and $\beta_{2} \leq d+\varepsilon$, hence $\lambda \geq d-\varepsilon$. Moreover $\operatorname{HD}(X) \geq 1-\varepsilon$. Hence

$$
\left|\left(f^{n}\right)^{\prime}\left(w_{n}\right)\right| \geq C \lambda_{n} \frac{(d-\varepsilon)^{\ell}}{(d+\varepsilon)^{\ell}} n^{-2\left(\frac{1}{(\ln (d-\varepsilon))(1-\varepsilon)}+\varepsilon\right) \ln d} \geq \lambda_{n} n^{-2-\varepsilon^{\prime}}
$$

with $\varepsilon$, hence $\varepsilon^{\prime}$, arbitrarily close to 0 . So, if $\lambda_{n} \geq C n^{3+\xi}$ the assumptions of Lemma 1 are satisfied and Theorem 1 follows.

QED

Remark 1 (corresponding to an observation in $[R]$ ). The measure of maximal entropy is optimal in this construction. If $\mu$ is any $f$-invariant ergodic measure on $J(f)$ of positive Lyapunov exponent $\chi_{\mu}(f):=\int \ln \left|f^{\prime}\right| d \mu$, then $(\ln \lambda) \operatorname{HD}(X) \approx \chi_{\mu}(f)\left(\mathrm{h}_{\mu}(f) / \chi_{\mu}(f)\right)=\mathrm{h}_{\mu}(f)$, where $\mathrm{h}_{\mu}(f)$ is the 
measure-theoretic entropy. $\approx$ means that the ratio is arbitrarily close to 1 for appropriate $X$. Therefore $\left|\left(f^{n}\right)^{\prime}\left(w_{n}\right)\right| \geq \lambda_{n} n^{-2 \ln d / \mathrm{h}_{\mu}(f)-\varepsilon^{\prime}}$, which attains maximum at $\mathrm{h}_{\mu}(f)=\mathrm{h}_{\text {top }}(f)=\ln d$, the topological entropy, giving (1).

Remark 2. The property $(*)$ excludes an existence of parabolic periodic points in $\mathrm{Fr} \Omega$. Otherwise we would find periodic orbits spending almost all the time close to such a parabolic point $q$, so its multiplier would about $C n^{a}$, where $a=t /(t-1) \leq 2$ for $f^{m}(z)=z+b(z-q)^{t}+\ldots$ for some integer $m$ and $b \neq 0$, for $z$ close to $q$.

The absence of Cremer periodic orbits follows from the local connectedness, see $[R]$ and the references there. We do not know whether Siegel discs can exist. The proof given in [PRS] under the assumption of the uniform exponential growth of the multipliers of repelling periodic orbits $\omega_{n}$ does not seem to work here. We do not know whether $\left(^{*}\right)$ implies a summability condition which would already imply the absence of Siegel discs and Cremer points due to so-called backward asymptotic stability, cf. [GS] or [P3, Th.B and Remark 3.2] and [PU2, Appendix B].

Now we pass to the setting of Theorem 2, where $R: \mathbb{D} \rightarrow \Omega$ is a Riemann mapping. Let $g$ be a holomorphic extension of $R^{-1} \circ f \circ R$ to a neighbourhood of the unit circle $\partial \mathbb{D}$. It exists and it is expanding on $\partial \mathbb{D}$, see $[\mathrm{P} 2, \S 7]$.

Now we formulate a lemma about the existence of appropriate expanding repellers. As we mentioned in Introduction it follows from Pesin-Katok theory. For the detailed proof see [P4], developing [PZ].

Lemma 3. Let $\nu$ be an ergodic $g$-invariant probability measure on $\partial \mathbb{D}$, such that for $\nu$-a.e. $\zeta \in \partial \mathbb{D}$ there exists a radial limit $\hat{R}(\zeta):=\lim _{r}{ }_{1} R(r \zeta)$. Assume that the measure $\mu:=\hat{R}_{*}(\nu)$ has positive Lyapunov exponent $\chi_{\mu}(f)$. Let $\varphi: \partial \mathbb{D} \rightarrow \mathbb{R}$ be a continuous real-valued function. Then for every $\varepsilon>0$ there exist $Y \subset \partial \mathbb{D}$ a $g$-invariant expanding repeller in the domain of $\hat{R}$ and $C>0$ such that for every $\delta>0$ small enough there exists $r(\delta)<1$, such that for all $r: r(\delta) \leq r<1$ and $\zeta \in Y$ and all positive integers $n$

(i) $C^{-1} \exp n\left(\int \varphi d \nu-\varepsilon\right) \leq \exp \sum_{j=0}^{n-1} \varphi\left(g^{j}(\zeta)\right) \leq C \exp n\left(\int \varphi d \nu+\varepsilon\right)$.

(ii) $X=\hat{R}(Y)$ is an expanding repeller for $f$ and for every $r: r(\delta)<$ $r<1$ it holds $R(r \zeta) \in B(\hat{R}(\zeta), \delta)$.

(iii) $C^{-1} \exp n\left(\chi_{\mu}(f)-\varepsilon\right) \leq\left|\left(f^{n}\right)^{\prime}(\hat{R}(\zeta))\right| \leq C \exp n\left(\chi_{\mu}(f)+\varepsilon\right)$.

(iv) $\operatorname{HD}(X) \geq \operatorname{HD}(\mu)-\varepsilon$. 
Proof of Theorem 2. For every $\zeta \in \partial \mathbb{I D}, \alpha: 0<\alpha<\pi / 2$ and $t>0$ denote

$$
S_{\alpha, t}(\zeta)=\zeta \cdot(1+\{x \in \mathbb{C} \backslash\{0\}: \pi-\alpha \leq \operatorname{Arg}(x) \leq \pi+\alpha\},|x|<t) .
$$

Such a set is called Stolz angle. If we do not mind $t$ we skip it and write $S_{\alpha}(\zeta)$.

By a distortion estimate for iterates of $g$ there exist $t_{0}<1, C>0$ and $\vartheta: 0<\vartheta<\pi / 2$ such that if for all $j=0,1,2, \ldots, m$ it holds $1-\left|g^{j}(r \zeta)\right| \leq t_{0}$ then $g^{m}(r \zeta) \in S_{\vartheta, C t_{0}}\left(g^{m}(\zeta)\right)$, for an arbitrary $m$.

Choose $X, Y$ and all the constants as in Lemma 3, with $\varphi=\ln \left|g^{\prime}\right|$. Consider an arbitrary positive integer $n$ and choose $\hat{x} \in X, \delta>0$ and $\ell$ as in Proof of Lemma 2, except that now $\ell$ is the first time $f^{\ell}\left(B\left(\hat{x}, \delta n^{-a}\right)\right.$ becomes large. (This $\ell$ was $k$ in Proof of Lemma 2.) We define only now $\hat{w}:=f^{\ell}(\hat{x})$. Therefore $\hat{w}$ depends on $n$.

Choose $y=s \hat{y}$ for $\hat{y} \in Y$ and $s: 0<s<1$, satisfying $\hat{R}(\hat{y})=\hat{x}$ such that $x:=R(y) \in \partial B\left(\hat{x}, \delta^{2} n^{-a}\right)$.

Note that in Proof of Th.1 $y$ was denoted by $\tilde{x}$. It was defined as $y=R^{-1}(x)$, after $x$ had been chosen. We did not care about the distance and position of $y$ with respect to $\hat{y}$. (The latter point was not of interest there, a priori we did not even know it existed.) Here we are more careful, consider $\hat{x}$ in the radial limit of a point $\hat{y}$ and choose $y$ belonging to the radius at $\hat{y}$.

If $\delta$ is small enough then all points $g^{j}(y)$ are close to $\partial \mathbb{D}$ for $j=0, \ldots, \ell$ since all the distances between $\hat{R} g^{j}(\hat{y})$ and $R g^{j}(y)$ are small, smaller than $C \delta$. (This is the reason why $\delta^{2}$ appears in the choice of $x$ ). Otherwise there would be a sequence of points in $\mathbb{D}$ with limit $z \in \mathbb{D}$ such that $R(z) \in \operatorname{Fr} \Omega$ by the continuity of $R$ in $\mathbb{D}$, which would contradict $R(\mathbb{D})=\Omega$.

In particular $g^{j}(y) \in S_{\vartheta, C t_{0}}\left(g^{j}(\hat{y})\right)$. So all the distances $\left|g^{j}(\hat{y})-g^{j}(y)\right|$ are small, hence by Lemma 3 (i) for $\zeta=\hat{y}$ and by the continuity of $\ln \left|g^{\prime}\right|$ we get

$$
\left|\left(g^{\ell}\right)^{\prime}(y)\right| \leq C \exp \ell\left(\chi_{\nu}(g)+2 \varepsilon\right)
$$

On the other hand the point $g^{\ell}(y) \in S_{\vartheta, C t_{0}}\left(g^{\ell}(\hat{y})\right)$ is well inside $\mathbb{D}$. This follows from the assumption that $w_{0}:=R\left(g^{\ell}(y)\right)=f^{\ell}(x) \in f^{\ell}\left(\partial B\left(\hat{x}, \delta^{2} n^{-a}\right)\right)$ is far from $f^{\ell}(\hat{x})$, namely within the distance at least $C \delta$.

This was the (only) place where we used the uniform radial continuity of $\hat{R}$ at $Y$ assured by Lemma 3 (ii); more precisely we used the uniform nontangential continuity of $R$, at $\zeta=g^{\ell}(\hat{y})$, namely the uniform convergence of $R(z)$ for $z \rightarrow \zeta$ such that $z \in S_{\vartheta}$. (Nontangential and radial convergences of $R$ are equivalent properties by a general theory). 
Then the final estimate in Proof of Theorem 1 replaces by

$$
\left|\left(f^{n}\right)^{\prime}\left(w_{n}\right)\right| \geq \lambda_{n} n^{-2 \chi_{\nu}(g) / \chi_{\mu}(f) \operatorname{HD}(\mu)-\varepsilon^{\prime}} .
$$

Now we apply $\operatorname{HD}(\mu)=\mathrm{h}_{\mu}(f) / \chi_{\mu}(f)$ see [PU1, Ch.9] and $\mathrm{h}_{\nu}(g)=\mathrm{h}_{\mu}(f)$, see $[\mathrm{P} 1]$ and $[\mathrm{P} 2, \S 4]$. We get

$$
\left|\left(f^{n}\right)^{\prime}\left(w_{n}\right)\right| \geq \lambda_{n} n^{-2 \chi_{\nu}(g) / \mathrm{h}_{\mu}(f)-\varepsilon^{\prime}}=\lambda_{n} n^{-2 \chi_{\nu}(g) / \mathrm{h}_{\nu}(g)-\varepsilon^{\prime}}=\lambda_{n} n^{-2-\varepsilon^{\prime}}
$$

the latter equality for $\nu$ equivalent to length (harmonic) measure, where $\chi_{\nu}(g) / \mathrm{h}_{\nu}(g)=\mathrm{HD}(\nu)=1$.

Though in this construction $w_{0}$ depends on $n$, this does not influence the result. We can replace at the end $w_{0}$ by a base point independent of $n$ which changes the final estimate only by a distortion constant, which can be absorbed by $\varepsilon^{\prime}$ for $n$ large enough.

QED

Remark 3. As in Remark 1 note that the measure $\nu$ equivalent to the length is optimal in the sense that for any other $g$-invariant probability measure of positive Lyapunov exponent (which implies that $\mu=\hat{R}_{*}(\nu)$ also has positive Lyapunov exponent, see [P2]), as $\operatorname{HD}(\nu) \leq \operatorname{HD}(\partial \mathbb{D})=1$, we obtain $\left|\left(f^{n}\right)^{\prime}\left(w_{n}\right)\right| \geq \lambda_{n} n^{\left.-2 \mathrm{HD}(\nu)-\varepsilon^{\prime}\right)}$, the estimate which is not better.

Remark 4. It would be natural to prove a local version of Theorem 2, in the setting of [P2], assuming $\left(^{*}\right)$ only for periodic orbits in $\mathrm{Fr} \Omega$. More precisely the question is whether the following holds:

Let $\Omega$ be a simply connected domain in $\overline{\mathbb{C}}$ and $f$ be a holomorphic map defined on a neighbourhood $W$ of $\operatorname{Fr} \Omega$ to $\overline{\mathbb{C}}$. Assume $f(W \cap \Omega) \subset \Omega$, $f(\operatorname{Fr} \Omega) \subset \operatorname{Fr} \Omega$ and $\operatorname{Fr} \Omega$ repells to the side of $\Omega$, that is $\bigcap_{n=0}^{\infty} f^{-n}(W \cap \bar{\Omega})=$ $\operatorname{Fr} \Omega$. Suppose that $\left(^{*}\right)$ holds for all repelling periodic points $p$ in $\operatorname{Fr} \Omega$. Then any Riemann map $R: \mathbb{D} \rightarrow \Omega$ extends continuously to $\bar{D}$ and $\operatorname{Fr} \Omega$ is locally connected.

We do not know how to overcome troubles with finding $N$ consecutive branches of $f^{-1}$ whose composition maps $F_{2}\left(B\left(\hat{x}, n^{-a}\right)\right)$ deep in $B^{\prime}$ (in the notation in Proof of Lemma 2). Even if we succeed we do not know whether the periodic point $p$ belongs to $\operatorname{Fr} \Omega$. The problem is that we want to control every backward branch of $f^{-n}$ leading $x$ into $\Omega$, rather than (measure) typical, as in [PZ], or in accordance to some invariant hyperbolic subset of $\mathrm{Fr} \Omega$.

Note that at least Lemma 3 holds in this setting, see [P4]. 


\section{References}

[1] J. Graczyk, S. Smirnov, Weak expansion and geometry of Julia sets, preprint 1999.

[2] F. Przytycki, J. Rivera-Letelier, S. Smirnov, Equivalence and topological invariance of conditions for non-uniform hyperbolicity in the iteration of rational maps, Inventiones Mathematicae 151.1, pp. 29-63, (2003).

[3] F. Przytycki, J. Rivera-Letelier, S. Smirnov, Equality of pressures for rational functions, Ergodic Theory and Dynamical Systems 23, pp. 891914, (2004).

[4] F. Przytycki, Hausdorff dimension of harmonic measure on the boundary of an attractive basin for a holomorphic map, Invent. Math. 80, pp. 161-179, (1985).

[5] F. Przytycki, Riemann map and holomorphic dynamics, Invent. Math. 85, pp. 439-455, (1986).

[6] F. Przytycki, Iterations of holomorphic Collet-Eckmann maps: Conformal and invariant measures. Appendix: On non-renormalizable quadratic polynomials, Transactions of the AMS 350.2, pp. 717-742, (1998).

[7] F. Przytycki, Expanding repellers in limit sets for iteration of holomorphic functions, Fundamenta Math. 186. 1, pp. 85-96, (2005).

[8] F. Przytycki, Hyperbolic Hausdorff dimension is equal to the minimal exponent of conformal measure on Julia set. A simple proof, Proceedings of Kyoto Conference, Feb. (2004).

[9] F. Przytycki, M. Urbański. Fractals in the Plane, Ergodic Theory Methods. to appear in Cambridge University Press. Available on http://www.math.unt.edu/ urbanski and http://www.impan.gov.pl/ feliksp

[10] F. Przytycki, M. Urbański, Porosity of Julia sets of non-recurrent and parabolic Collet-Eckmann rational functions, Annales Academiae Scientiarum Fennicae 26, pp. 125-154, (2001). 
[11] F. Przytycki, A. Zdunik, Density of periodic sources in the boundary of a basin of attraction for iteration of holomorphic maps: geometric coding trees techniques, Fundamenta Math. 145, pp. 65-77, (1994).

[12] J. Rivera-Letelier. Weak hyperbolicity on periodic orbits for polynomials, C. R. Acad. Sci. Paris 334, pp. 1113-1118, (2002).

\section{Feliks Przytycki}

Institute of Mathematics

Polish Academy of Sciences

ul. Śniadeckich 8

00-956 Warszawa

Poland

e-mail: feliksp@impan.gov.pl 